Supplement of Biogeosciences, 18, 95-112, 2021 https://doi.org/10.5194/bg-18-95-2021-supplement (C) Author(s) 2021. This work is distributed under the Creative Commons Attribution 4.0 License.

(c) (1)

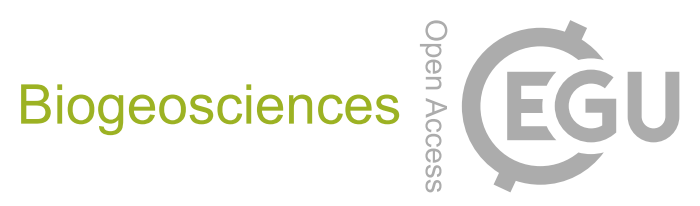

Supplement of

\title{
Improving the representation of high-latitude vegetation distribution in dynamic global vegetation models
}

Peter Horvath et al.

Correspondence to: Peter Horvath (peter.horvath@nhm.uio.no)

The copyright of individual parts of the supplement might differ from the CC BY 4.0 License. 


\section{Supplement S1 - Locations of 20 study plots}

Table S1 - Centre coordinates (latitude and longitude) and climatic data for the 20 plots used in this study. Estimates of mean annual precipitation and mean annual temperature are obtained from two sources; data from seNorge (C. Lussana et al., 2018; Lussana, Tveito, \& Uboldi, 2018) interpolated to each centre point and from CORDEX (the forcing climate dataset in DGVM).

\begin{tabular}{|l|c|c|c|c|c|c|c|c|}
\hline & & & & & \multicolumn{2}{c|}{$\begin{array}{c}\text { seNorge v2 data } \\
\text { (used in DM) }\end{array}$} & \multicolumn{2}{c|}{$\begin{array}{c}\text { CORDEX climate data } \\
\text { (used in DGVM) }\end{array}$} \\
\hline ID & $\begin{array}{c}\text { Plot \# from } \\
(\text { AR18×18) }\end{array}$ & LAT & LONG & $\begin{array}{c}\text { Elevation } \\
\text { (m a.s.1) } \\
\text { at centre }\end{array}$ & $\begin{array}{c}\text { Mean Annual } \\
\text { Precipitation } \\
(\mathrm{mm})\end{array}$ & $\begin{array}{c}\text { Mean Annual } \\
\text { Temperature } \\
\left({ }^{\circ} \mathrm{C}\right)\end{array}$ & $\begin{array}{c}\text { Mean Annual } \\
\text { Precipitation } \\
(\mathrm{mm})\end{array}$ & $\begin{array}{c}\text { Mean Annual } \\
\text { Temperature } \\
\left({ }^{\circ} \mathrm{C}\right)\end{array}$ \\
\hline 3 & 405 & 6.061 & 58.635 & 200 & 2662 & 6.3 & 2916 & 4.7 \\
\hline 2 & 513 & 6.035 & 59.934 & 710 & 2628 & 1.0 & 3530 & 2.9 \\
\hline 1 & 622 & 5.956 & 61.392 & 596 & 2520 & 2.0 & 2606 & 2.0 \\
\hline 6 & 801 & 7.429 & 58.074 & 184 & 1542 & 6.7 & 2055 & 5.9 \\
\hline 4 & 922 & 6.957 & 61.456 & 1437 & 1799 & -3.6 & 2958 & -2.9 \\
\hline 5 & 1131 & 7.264 & 62.935 & 454 & 1976 & 4.0 & 1716 & 4.8 \\
\hline 8 & 1304 & 8.862 & 58.638 & 88 & 1395 & 7.1 & 1640 & 4.9 \\
\hline 7 & 1322 & 8.298 & 61.529 & 1670 & 827 & -3.1 & 2418 & -6.1 \\
\hline 9 & 1623 & 9.278 & 61.735 & 852 & 555 & -0.1 & 808 & -3.9 \\
\hline 10 & 2015 & 10.812 & 60.496 & 606 & 804 & 1.9 & 1517 & 0.5 \\
\hline 12 & 2108 & 11.268 & 59.377 & 130 & 1072 & 5.5 & 1223 & 4.4 \\
\hline 11 & 2238 & 11.000 & 64.223 & 222 & 1349 & 4.3 & 1542 & 2.1 \\
\hline 13 & 2332 & 11.492 & 63.266 & 721 & 1029 & 0.3 & 2001 & -0.2 \\
\hline 14 & 2425 & 11.968 & 62.145 & 744 & 715 & -1.2 & 1013 & -2.0 \\
\hline 16 & 2948 & 13.508 & 65.886 & 529 & 1513 & 1.1 & 1819 & -0.3 \\
\hline 15 & 2962 & 13.363 & 68.146 & 393 & 1339 & 5.8 & 1075 & 4.4 \\
\hline 17 & 4268 & 19.167 & 69.072 & 354 & 715 & 0.7 & 1122 & -1.8 \\
\hline 18 & 5369 & 24.147 & 69.040 & 395 & 466 & -4.0 & 695 & -3.1 \\
\hline 19 & 6473 & 29.382 & 69.334 & 69 & 503 & -1.1 & 640 & -2.5 \\
\hline 20 & 6380 & 29.703 & 70.465 & 387 & 552 & 0.2 & 1132 & -2.5 \\
\hline
\end{tabular}




\section{Supplement S2 - Assessment of climatic representativeness of selected plots}

We assessed the representativeness of the 20 plots, selected from the original AR18 $\times 18$ dataset which consists of 1081 plots, by comparing frequency distributions with respect to the two main bioclimatic gradients in Norway, expressed as annual mean temperature and annual precipitation. We also included a comparison of precipitation seasonality, as the only one of the three tested new parameters that improved the DGVM in the sensitivity tests. For each of temperature, precipitation and precipitation seasonality, we obtained values for the centre point of each AR18×18 plot (cf. Fig. S1) and compared the frequency distributions of the selected plots with those of all plots (Fig. S3). A series of Kolmogorov-Smirnov tests for these three variables (comparison of sample mean and variance) indicate that the subsample does not deviate from the full dataset substantially. The 20 selected plots span elevations from 88 to $1670 \mathrm{~m}$ a.s.l., covers an annual temperature range from $-4^{\circ} \mathrm{C}$ to $7.1^{\circ} \mathrm{C}$, and an annual precipitation range from 466 to $2661 \mathrm{~mm}$ (Fig. S1), which accords well with the variation in the AR18×18 dataset (Fig. S2).
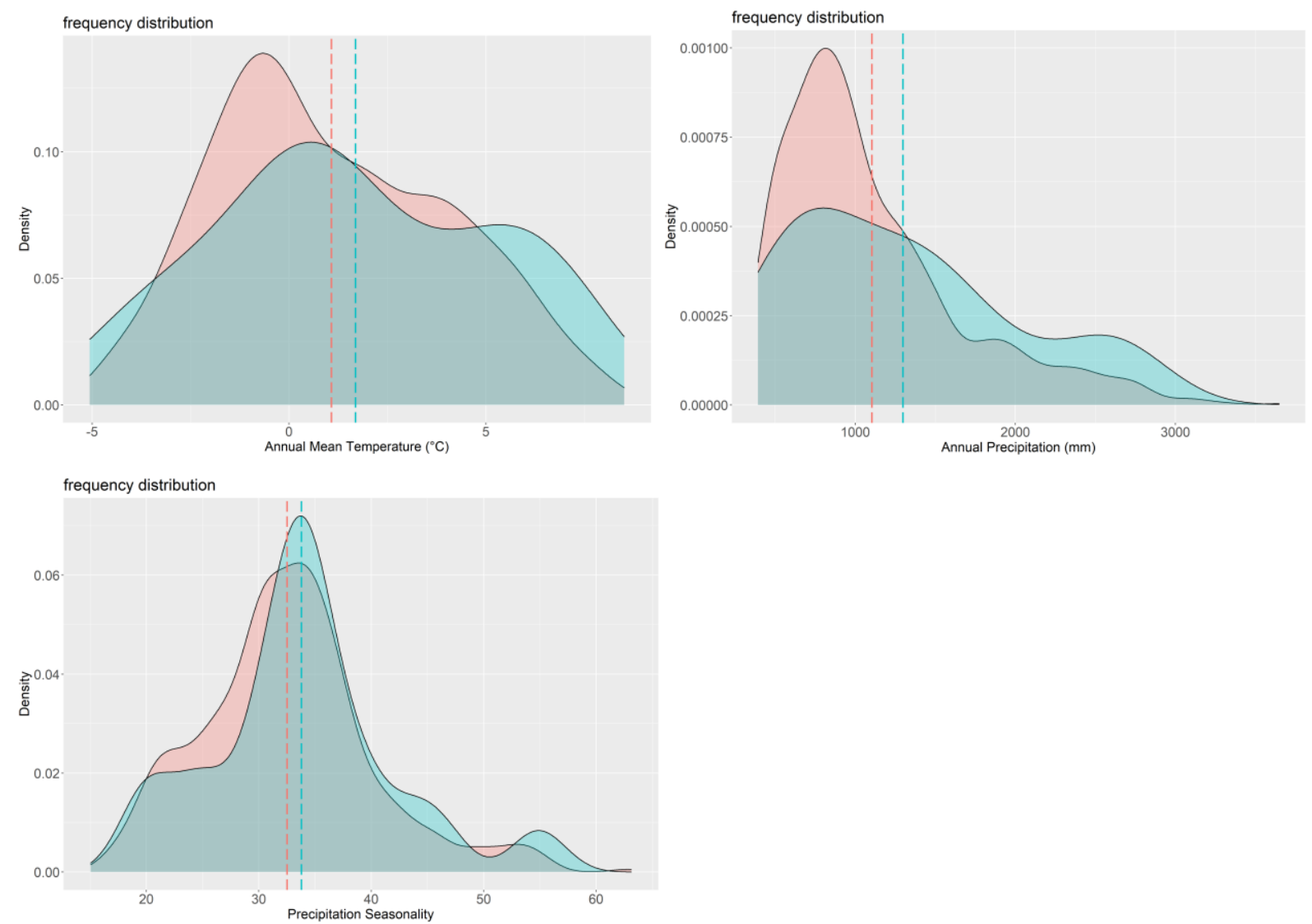

Figure S2- Frequency distributions of plots in the original AR18 $\times 18$ dataset $(\mathrm{n}=1081$; in red) and in the set of 20 plots selected for this study (in blue), with respect to annual mean temperature (top left),annual precipitation (top right) and precipitation seasonality (bottom left). Dashed lines indicate means for the respective datasets. 


\section{Supplement S3 - Assessment of the representativeness of PFT profiles}

We also assessed the representativeness of the 20 study plots, selected from the original AR18×18 dataset which consists of 1081 plots, by comparing the aggregated PFT profiles for the two datasets given in Table S4. PFT profiles were first obtained for each plot by the conversion scheme in Table S5, thereafter aggregated to dataset level by calculation of mean frequencies for each of the six PFTs (and 'EXCL'; land not assigned to any PFT type).

The comparison between the aggregated PFT profiles in Table S4 by use of the chi-square test (see section 2.6 for method) shows that the two datasets are much more similar than expected by chance $\left(\chi^{2}=1.991, \mathrm{df}=6, \mathrm{p}=0.079\right)$. Despite slight overrepresentation of the boreal NET PFT and underrepresentation of boreal BDT and C3 grasses, we conclude that the selected plots are sufficiently representative for the conclusions drawn from the sample of 20 plots to be acceptably representative for Norway. Note that percentage for EXCL category has been proportionally re-distributed through relevant PFTs in the study as shown on the Table S3 (so that the six PFTs cover 100\%).

Table S3 - PFT profiles of the full AR18×18 dataset $(n=1081)$ and the 20 plots selected for this study.

\begin{tabular}{|l|l|l|l|}
\hline PFT code & PFT name & $\begin{array}{l}\text { Fraction of PFT in } \\
1081 \text { plots }(\%)\end{array}$ & $\begin{array}{l}\text { Fraction of PFT in 20 } \\
\text { plots (\%) }\end{array}$ \\
\hline BG & Bare Ground & 10.37 & 10.95 \\
\hline Boreal NET & needleleaf evergreen tree - boreal & 21.50 & 31.18 \\
\hline Temp BDT & broadleaf deciduous tree - temperate & 0.46 & 0.40 \\
\hline Boreal BDT & broadleaf deciduous tree - boreal & 16.02 & 12.55 \\
\hline Boreal BDS & broadleaf deciduous shrub - boreal & 25.11 & 24.35 \\
\hline C3 & C3 grass & 7.27 & 3.00 \\
\hline EXCL & excluded & 19.27 & 17.57 \\
\hline
\end{tabular}




\section{Supplement S4 - Assessment of the representativeness of climate forcing data}

The comparison of seNorge and CORDEX estimates of temperature and precipitation in Fig. S5.1 shows that precipitation estimates by CORDEX for the 20 plots were generally higher than seNorge estimates while the converse (but less strongly) was true for temperature.

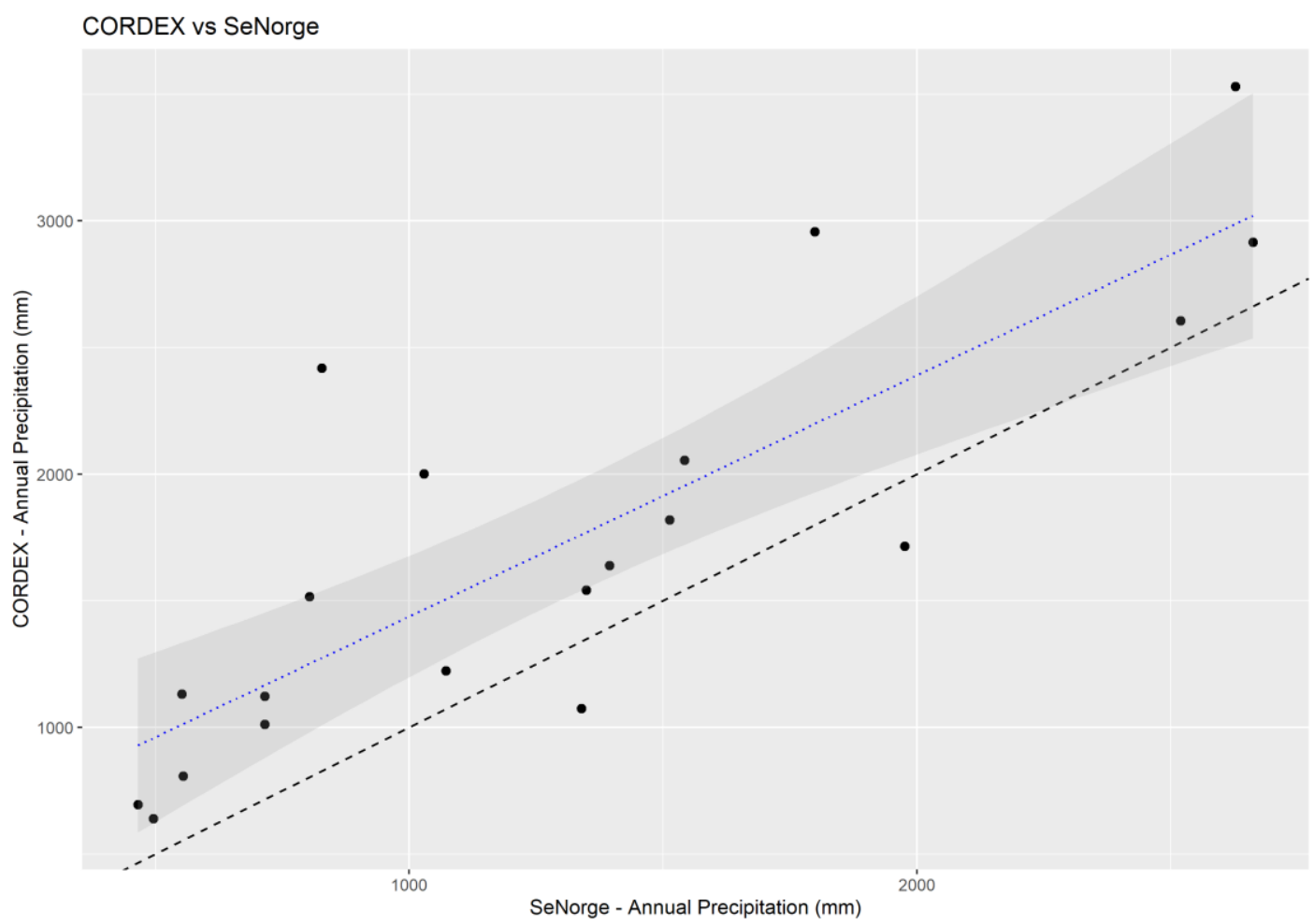

CORDEX vs SeNorge

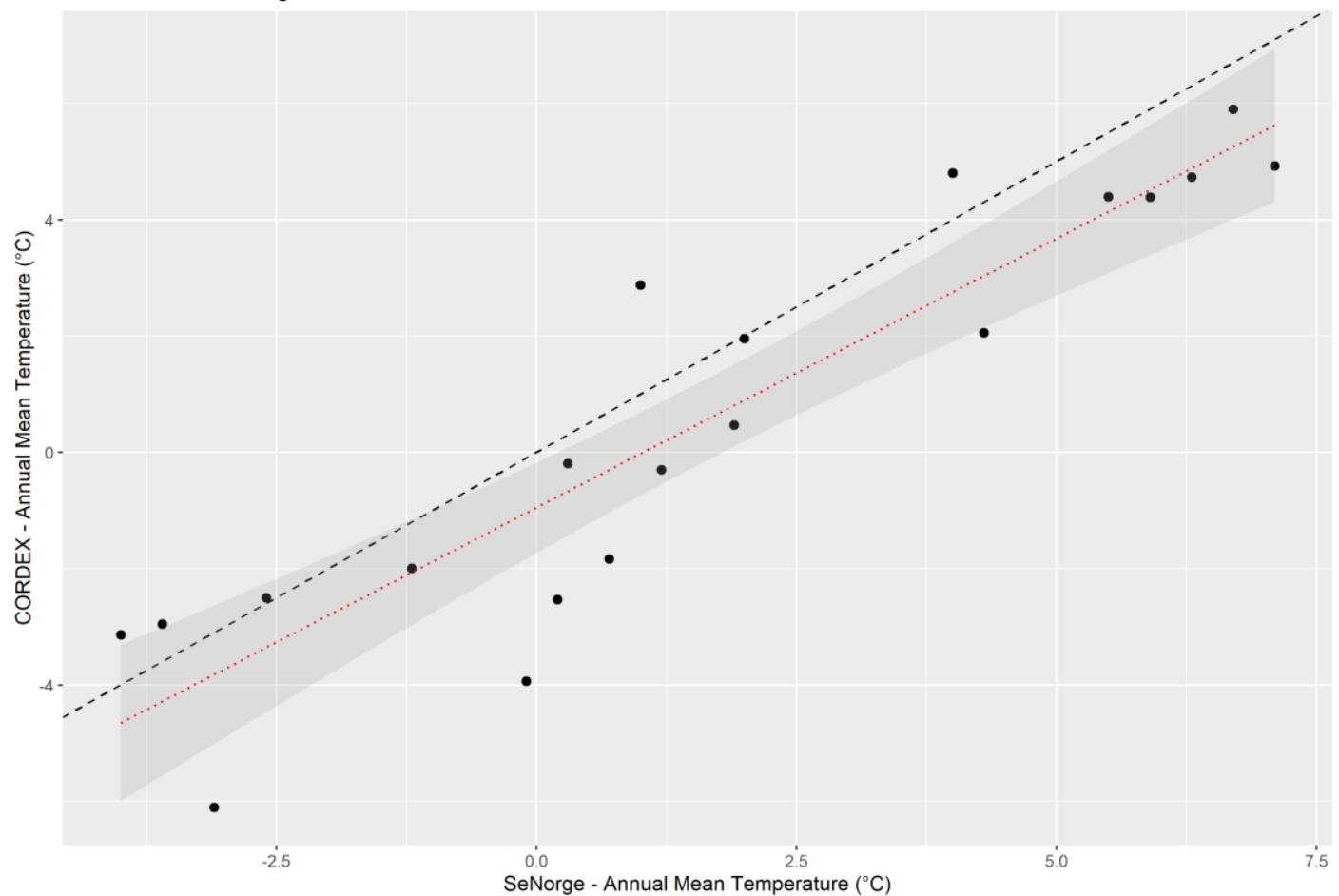

Figure S4 - Scatterplots showing the relationship between temperature and precipitation estimates obtained by the two data sources used in this study; seNorge for DM (see Sect. 2.4.3) on the horizontal axes and CORDEX for climate forcing data used in DGVM (see Sect. 2.4.1) on the vertical axis. The dashed black line represents the 1:1 relationship, while the dotted red line represents a linear model of $y \sim x$. 
Supplement S5 - PFT Conversion scheme

Table S5- Conversion scheme for harmonizing vegetation and land cover types across methods (RS, DM and AR) into plant functional types (PFTs). DGVM - dynamic global vegetation model, RS - remote sensing, DM - distribution model, AR - reference dataset. PFT - plant functional type and VT - vegetation type.

\begin{tabular}{|c|c|c|c|c|}
\hline \multicolumn{2}{|l|}{ DGVM } & \multirow{2}{*}{\begin{tabular}{|l|} 
RS \\
vegetation / land cover type \\
- remote sensing
\end{tabular}} & \multirow{2}{*}{$\begin{array}{l}\text { DM } \\
\text { vegetation type - distribution } \\
\text { model }\end{array}$} & \multirow{2}{*}{\begin{tabular}{|l|}
$\mathrm{AR}$ \\
$\begin{array}{l}\text { vegetation type - area frame } \\
\text { survey }\end{array}$
\end{tabular}} \\
\hline PFT & $\begin{array}{l}\text { plant functional } \\
\text { type }\end{array}$ & & & \\
\hline \multirow{7}{*}{ BG } & \multirow{7}{*}{ Bare ground } & $\begin{array}{l}\text { Exposed alpine ridges, scree } \\
\text { and rock complex }\end{array}$ & Frozen ground, leeward & Frozen ground, leeward \\
\hline & & & Frozen ground, ridge & Frozen ground, ridge \\
\hline & & & Boulder field & Sand dunes and gravel beaches \\
\hline & & & Exposed bedrock & Pioneer alluvial vegetation \\
\hline & & & & Barren land \\
\hline & & & & Boulder field \\
\hline & & & & Exposed bedrock \\
\hline \multirow{9}{*}{$\begin{array}{l}\text { Boreal } \\
\text { NET }\end{array}$} & \multirow{9}{*}{$\begin{array}{l}\text { Boreal } \\
\text { needleleaf } \\
\text { evergreen tree }\end{array}$} & $\begin{array}{l}\text { Coniferous forest }- \text { dense } \\
\text { canopy layer }\end{array}$ & $\begin{array}{l}\text { Lichen and heather pine } \\
\text { forest }\end{array}$ & Lichen and heather pine forest \\
\hline & & $\begin{array}{l}\text { Coniferous forest and mixed } \\
\text { forest - open canopy }\end{array}$ & Bilberry pine forest & Bilberry pine forest \\
\hline & & Lichen rich pine forest & $\begin{array}{l}\text { Lichen \& heather spruce } \\
\text { forest }\end{array}$ & Meadow pine forest \\
\hline & & & Bilberry spruce forest & Pine forest on lime soils \\
\hline & & & Meadow spruce forest & Lichen \& heather spruce forest \\
\hline & & & Damp forest & Bilberry spruce forest \\
\hline & & & Bog forest & Meadow spruce forest \\
\hline & & & & Damp forest \\
\hline & & & & Bog forest \\
\hline \multirow{2}{*}{$\begin{array}{l}\text { Temperate } \\
\text { BDT }\end{array}$} & \multirow{2}{*}{$\begin{array}{l}\text { Temperate } \\
\text { broadleaf } \\
\text { deciduous tree }\end{array}$} & $\begin{array}{l}\text { Low herb forest and } \\
\text { broadleaved } \\
\text { forest }\end{array}$ & $\begin{array}{l}\text { Poor / Rich broadleaf } \\
\text { deciduous forest }\end{array}$ & $\begin{array}{l}\text { Poor broadleaf deciduous } \\
\text { forest }\end{array}$ \\
\hline & & & & $\begin{array}{l}\text { Rich broadleaf deciduous } \\
\text { forest }\end{array}$ \\
\hline \multirow{8}{*}{$\begin{array}{l}\text { Boreal } \\
\text { BDT }\end{array}$} & \multirow{8}{*}{$\begin{array}{l}\text { Boreal } \\
\text { broadleaf } \\
\text { deciduous tree }\end{array}$} & $\begin{array}{l}\text { Tall herb - tall fern } \\
\text { deciduous forest }\end{array}$ & $\begin{array}{l}\text { Lichen and heather birch } \\
\text { forest }\end{array}$ & Lichen and heather birch forest \\
\hline & & $\begin{array}{l}\text { Bilberry- low fern birch } \\
\text { forest }\end{array}$ & Bilberry birch forest & Bilberry birch forest \\
\hline & & Crowberry birch forest & Meadow birch forest & Meadow birch forest \\
\hline & & Lichen-rich birch forest & Alder forest & Birch forest on lime soils \\
\hline & & & Pasture land forest & Alder forest \\
\hline & & & Poor / rich swamp forest & Pasture land forest \\
\hline & & & & Poor swamp forest \\
\hline & & & & Rich swamp forest \\
\hline \multirow{10}{*}{$\begin{array}{l}\text { Boreal } \\
\text { BDS }\end{array}$} & \multirow{10}{*}{$\begin{array}{l}\text { Boreal } \\
\text { broadleaf } \\
\text { deciduous shrub }\end{array}$} & $\begin{array}{l}\text { Heather-rich alpine ridge } \\
\text { vegetation }\end{array}$ & Lichen heath & Lichen heath \\
\hline & & Lichen-rich heathland & Mountain avens heath & Mountain avens heath \\
\hline & & $\begin{array}{l}\text { Heather- and grass-rich early } \\
\text { snow patch communities }\end{array}$ & $\begin{array}{l}\text { Dwarf shrub / Alpine calluna } \\
\text { heath }\end{array}$ & Dwarf shrub heath \\
\hline & & $\begin{array}{l}\text { Fresh heather and dwarf- } \\
\text { shrub communities (u/l) }\end{array}$ & Alpine damp heath & Alpine calluna heath \\
\hline & & & $\begin{array}{l}\text { Coastal heath / Coastal } \\
\text { calluna heath }\end{array}$ & Alpine damp heath \\
\hline & & & Damp heath & Flood-plain shrubs \\
\hline & & & & Coastal heath \\
\hline & & & & Coastal calluna heath \\
\hline & & & & Damp heath \\
\hline & & & & Crags and thicket \\
\hline \multirow{4}{*}{$\mathrm{C} 3$} & \multirow{4}{*}{$\mathrm{C} 3$ grass } & $\begin{array}{l}\text { Graminoid alpine ridge } \\
\text { vegetation }\end{array}$ & $\begin{array}{l}\text { Moss snowbed / Sedge and } \\
\text { grass snowbed }\end{array}$ & Moss snowbed \\
\hline & & $\begin{array}{l}\text { Herb-rich meadows (up- } \\
\text { /lowland) }\end{array}$ & Dry grass heath & Sedge and grass snowbed \\
\hline & & $\begin{array}{l}\text { Grass and dwarf willow } \\
\text { snow-patch vegetation }\end{array}$ & Low herb / forb meadow & Dry grass heath \\
\hline & & & & Low herb meadow \\
\hline
\end{tabular}




\begin{tabular}{|c|c|c|c|c|}
\hline & & & & Low forb meadow \\
\hline & & & & Moist and shore meadows \\
\hline \multirow{13}{*}{ EXCL } & \multirow{13}{*}{ Excluded } & $\begin{array}{l}\text { Ombrotrophic bog and low- } \\
\text { grown swamp vegetation }\end{array}$ & $\begin{array}{l}\text { Bog / Mud-bottom fen and } \\
\text { bog }\end{array}$ & Bog \\
\hline & & $\begin{array}{l}\text { Tall-grown } \quad \text { swamp } \\
\text { vegetation }\end{array}$ & Deer-grass fen / fen & Deer-grass fen \\
\hline & & $\begin{array}{l}\text { Wet mires, sedge swamps } \\
\text { and reed beds }\end{array}$ & Sedge marsh & Fen \\
\hline & & $\begin{array}{l}\text { Glacier, snow and wet snow- } \\
\text { patch vegetation }\end{array}$ & Pastures & Mud-bottom fen and bog \\
\hline & & Water & & Sedge marsh \\
\hline & & Agricultural areas & & Cultivated land \\
\hline & & Cities and built-up areas & & Pastures \\
\hline & & $\begin{array}{l}\text { Unclassified and shadow } \\
\text { affected areas, }\end{array}$ & & Built-up areas \\
\hline & & & & Scattered housing \\
\hline & & & & Artificial impediment \\
\hline & & & & Glaciers and perpetual snow \\
\hline & & & & Sea and ocean \\
\hline & & & & Water bodies (fresh) \\
\hline
\end{tabular}


Supplement S6 - Sampling design - RS, DM and AR

\section{RS - Remote sensing $\quad$ DM - Distribution model $\quad$ AR - Reference dataset}

plot 7
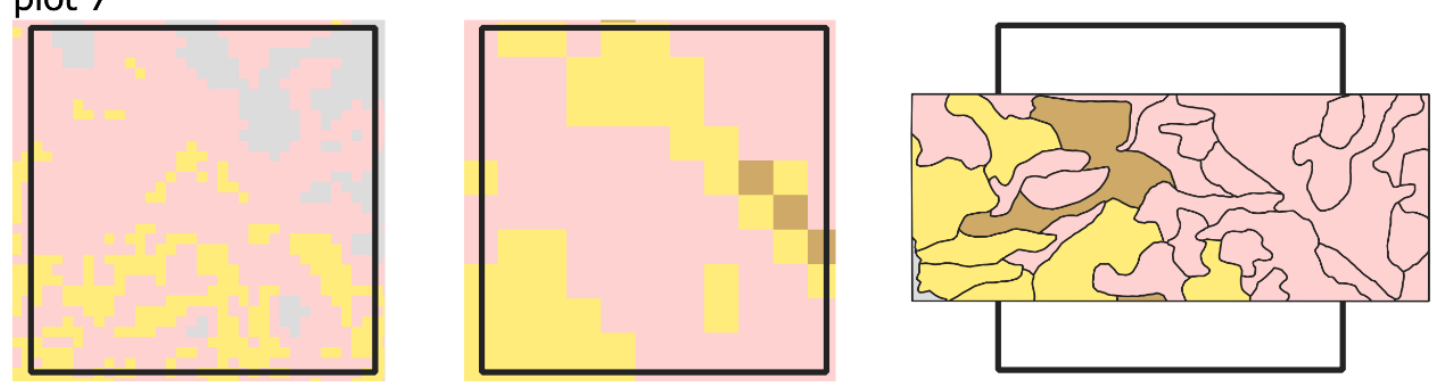

plot 14
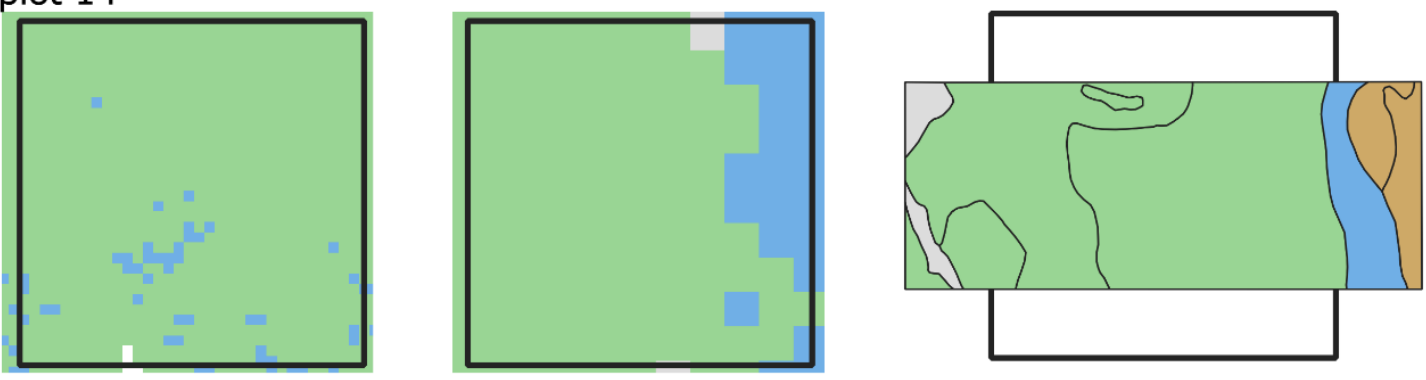

Plant Functional Types

$\square$ BG - bare ground

boreal NET - needleleaf evergreen boreal tree

temperate BDT - broadleaf deciduous temperate tree

boreal BDT - broadleaf deciduous boreal tree

boreal BDS - broadleaf deciduous boreal shrub

C3 - C3 Grass

excluded

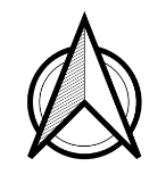

Figure S6 - Sampling design used by the remote sensing (RS) and distribution modelling (DM) methods and to obtain the AR reference dataset. Like DGVM plots (see Fig. S7), the RS and DM plots are $1 \times 1 \mathrm{~km}$, while the AR plots are $1.5 \times 0.6 \mathrm{~km}$. Plots 7 and plot 14 (AR18×18 plot \#1322 and plot \#2425) are used as examples. 


\section{Supplement S7 - DGVM parameters for PFTs (CLM4.5-BGCDV)}

Table S7 - Some important PFT parameter settings for DGVM (CLM4.5-DV). PFTs relevant for the study area (Norway) are shaded grey. Prescribed heights for the canopy are indicated by the upper and lower limits in columns "ztop" and "zbot" respectively. Limiting temperatures for survival and establishment are mentioned in columns "Tc,min" and "Tc,max" respectively. Minimum growing degree days for establishment are contained for relevant PFTs in column "GDDmin". The last three columns contain the new parameter thresholds used in the sensitivity experiment. swe_10 - snow water equivalent in October $(\mathrm{mm})$; tmin_5 - minimum temperature in May $\left({ }^{\circ} \mathrm{C}\right)$ bioclim_15 - precipitation seasonality (coefficient of variation).

\begin{tabular}{|c|c|c|c|c|c|c|c|c|c|}
\hline \multirow[b]{2}{*}{ Plant functional type (PFT) } & \multirow[b]{2}{*}{ Acronym } & \multicolumn{2}{|c|}{ Prescribed heights } & \multirow{2}{*}{$\begin{array}{l}\text { Survival } \\
\text { Tc,min } \\
\left({ }^{\circ} \mathrm{C}\right)\end{array}$} & \multicolumn{2}{|c|}{ Establishment } & \multicolumn{3}{|c|}{ Sensitivity tests } \\
\hline & & ztop (m) & zbot $(\mathrm{m})$ & & $\begin{array}{l}\text { Tc,max } \\
\left({ }^{\circ} \mathrm{C}\right)\end{array}$ & GDDmin & $\begin{array}{l}\begin{array}{l}\text { swe_10 } \\
(\mathrm{mm})\end{array} \\
\end{array}$ & $\begin{array}{l}\text { tmin_5 } \\
\left({ }^{\circ} \mathrm{C}\right)\end{array}$ & bioclim_15 \\
\hline Needleleaf evergreen tree - temperate & Temp NET & 17 & 8.5 & -2 & 22 & 900 & & & \\
\hline Needleleaf evergreen tree - boreal & Boreal NET & 17 & 8.5 & -32.5 & -2 & 600 & 150 & -5 & 50 \\
\hline Needleleaf deciduous tree - boreal & Boreal NDT & 14 & 7 & & & & & & \\
\hline Broadleaf evergreen tree - tropical & Trop BET & 35 & 1 & 15.5 & No limit & 0 & & & \\
\hline Broadleaf evergreen tree - temperate & Temp BET & 35 & 1 & 3 & 18.8 & 1200 & & & \\
\hline Broadleaf deciduous tree - tropical & Trop BDT & 18 & 10 & 15.5 & No limit & 0 & & & \\
\hline Broadleaf deciduous tree - temperate & Temp BDT & 20 & 11.5 & -17 & 15.5 & 1200 & & & \\
\hline Broadleaf deciduous tree - boreal & Boreal BDT & 20 & 11.5 & No limit & -2 & 350 & 180 & -7.5 & \\
\hline Broadleaf evergreen shrub - temperate & Temp BES & 0.5 & 0.1 & & & & & & \\
\hline Broadleaf deciduous shrub - temperate & Temp BDS & 0.5 & 0.1 & -17 & No limit & 1200 & & & \\
\hline Broadleaf deciduous shrub - boreal & Boreal BDS & 0.5 & 0.1 & No limit & -2 & 350 & 380 & -10 & \\
\hline $\mathrm{C} 3$ arctic grass & C3 A & 0.5 & 0.01 & No limit & -17 & 0 & & & \\
\hline $\mathrm{C} 3$ grass & $\mathrm{C} 3$ & 0.5 & 0.01 & -17 & 15.5 & 0 & & & \\
\hline $\mathrm{C} 4$ grass & $\mathrm{C} 4$ & 0.5 & 0.01 & 15.5 & No limit & 0 & & & \\
\hline Non vegetated/bare ground & BG & & & & & & & & \\
\hline
\end{tabular}


Supplement S8 - Representation of grid-cells in the CLM 4.5 model

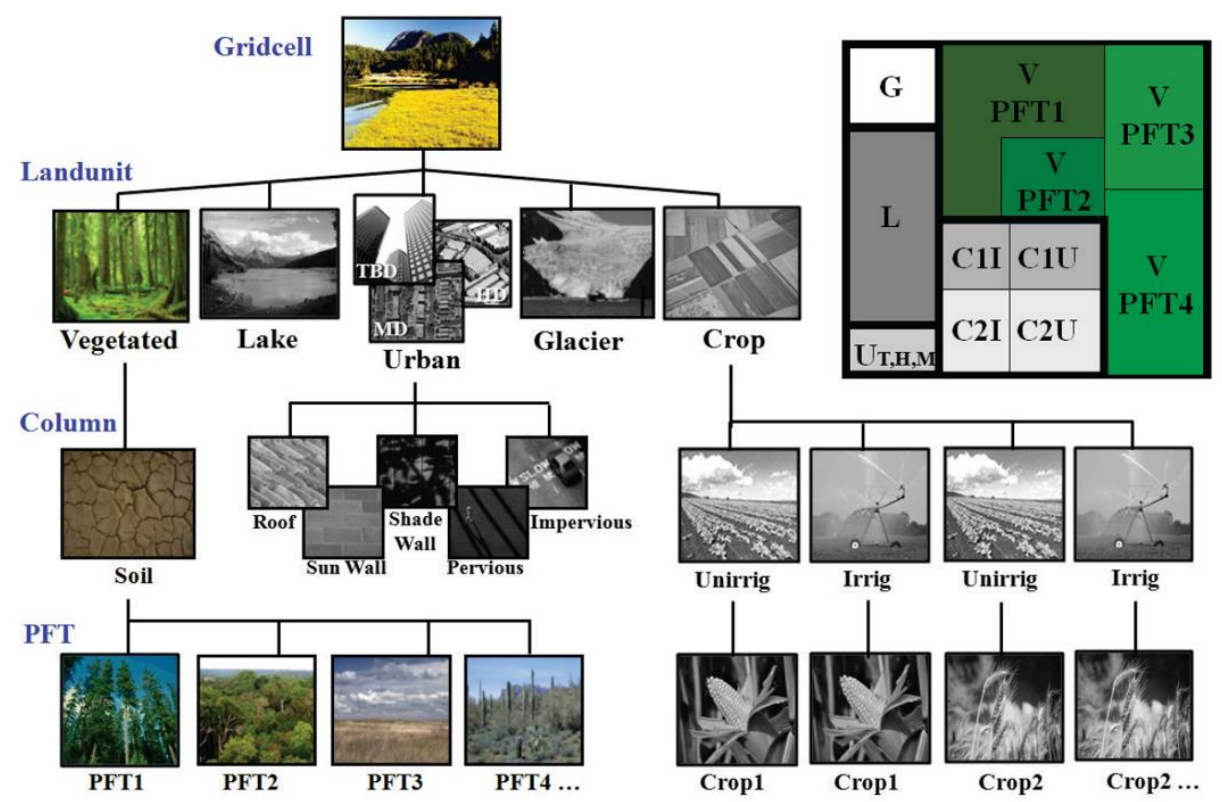

Figure S8 - Representation of a grid-cell in the DGVM model (obtained by CLM4.5-BGCDV method); figure adapted from Oleson et al. (2013). Land units in grey (lake, urban, glacier and crop) were excluded from this study. 


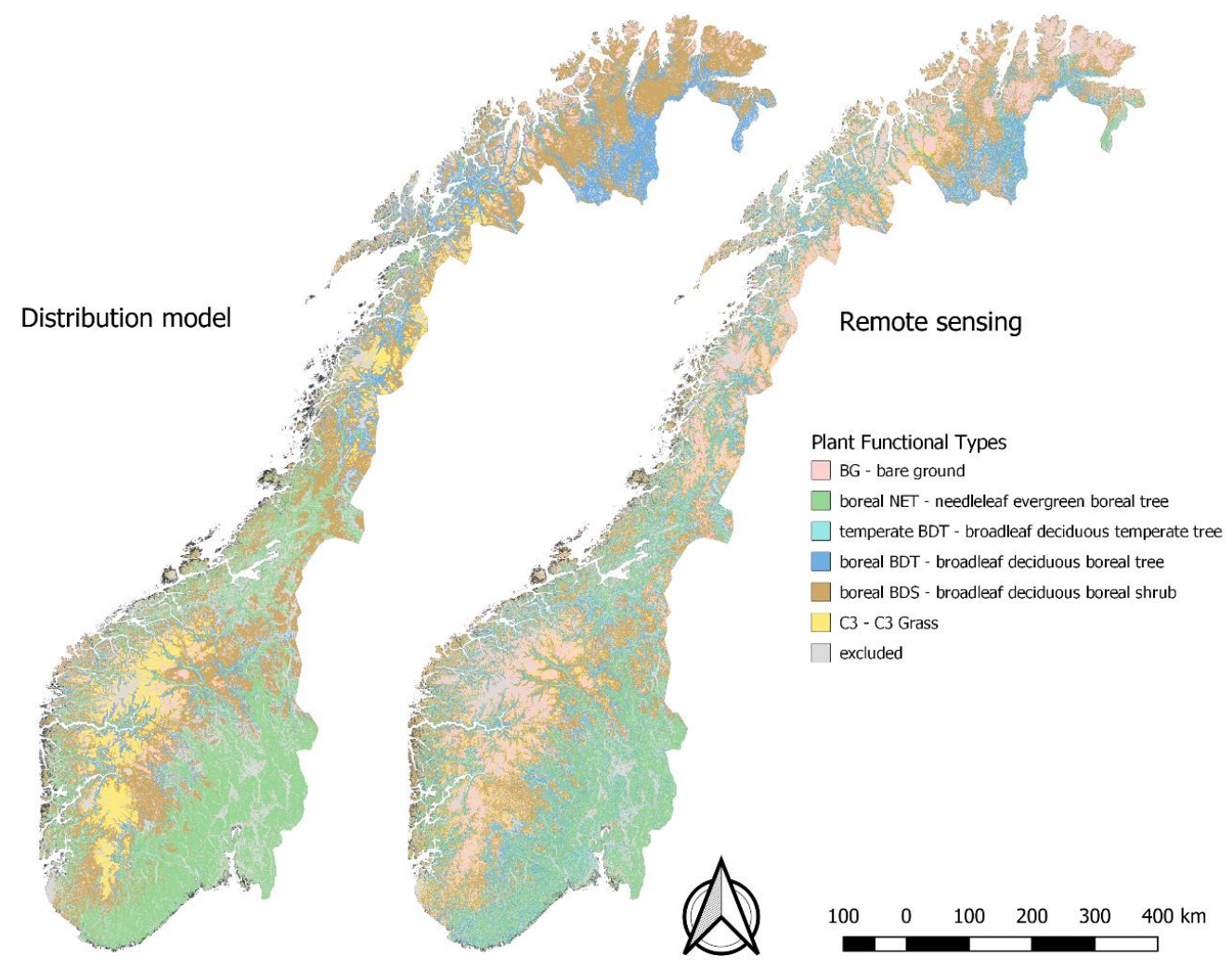

Figure S9- The distribution in Norway of vegetation types (used in distribution modelling - DM) and units obtained by remote sensing (RS), after reclassification to PFT units (see Table S5 for conversion scheme and explanation of PFT codes). The dominating PFT in each grid cell (of $100 \times 100 \mathrm{~m}$ for DM and $30 \times 30 \mathrm{~m}$ for RS) is shown.

The distributions in Norway of PFTs obtained by conversion of DM- and RS-units using the conversion scheme in Table S5 exhibit considerable similarities (Fig. S8). Both methods show dominance of boreal needleleaf evergreen forest (boreal NET) in south-eastern Norway, while most of the western and northern Norway is covered by boreal broadleaf deciduous shrub (boreal BDS) and boreal broadleaf deciduous forest (boreal BDT). Slight differences between the two methods can be seen in the western mountainous part of Norway, where DM predicts dominance by C3 grasses where RS suggests bare ground, and in North Norway where DM predicts boreal BDS where RS predicts bare ground. Accordingly, the fractional area classified to PFTs that are converted to bare ground is three times higher with RS than with DM (Table S8). Full resolution raster images are available at the Dryad repository (https://doi.org/10.5061/dryad.dfn2z34xn).

Table S9 - Area statistics for Norway for vegetation types (used in distribution modelling - DM) and units obtained by remote sensing (RS), after reclassification to PFT units (see Table S5 for conversion scheme and explanation of PFT codes).

\begin{tabular}{|l|l|l|}
\hline & RS (\%) & DM (\%) \\
\hline BG & 17.1 & 5.6 \\
\hline Boreal NET & 25.3 & 31.4 \\
\hline Temperate BDT & 5.2 & 0.1 \\
\hline Boreal BDT & 16.9 & 15.0 \\
\hline Boreal BDS & 27.9 & 39.0 \\
\hline C3 & 7.5 & 8.9 \\
\hline
\end{tabular}




\section{Supplement S10 - PFT profiles for each of the 20 plots}

Table S10- PFT profiles (percentage of vegetated land assigned to each of six PFTs) for each of the 20 plots in this study, obtained by remote sensing (RS) and distribution modelling (DM) methods and for the AR reference dataset. Original units (vegetation types, etc.) are converted to PFTs by use of the scheme in Table S5.

\begin{tabular}{|c|c|c|c|c|c|c|c|c|c|c|c|c|c|c|c|c|c|c|c|c|c|}
\hline Method & PFT shortcut & plot 3 & plot 2 & plot 1 & plot 6 & plot 4 & plot 5 & plot 8 & plot 7 & plot 9 & plot 10 & plot 12 & plot 11 & plot 13 & plot 14 & plot 16 & plot 15 & plot 17 & plot 18 & plot 19 & plot 20 \\
\hline DGVM & BG & 5 & 6 & 5 & 0 & 100 & 6 & 5 & 100 & 5 & 5 & 0 & 5 & 100 & 5 & 100 & 5 & 28 & 5 & 100 & 5 \\
\hline DGVM & boreal NET & 29 & 58 & 95 & 39 & 0 & 52 & 95 & 0 & 95 & 95 & 41 & 95 & 0 & 95 & 0 & 92 & 72 & 95 & 0 & 95 \\
\hline DGVM & temp. BDT & 35 & 2 & 0 & 34 & 0 & 4 & 0 & 0 & 0 & 0 & 38 & 0 & 0 & 0 & 0 & 0 & 0 & 0 & 0 & 0 \\
\hline DGVM & boreal BDT & 18 & 2 & 0 & 22 & 0 & 4 & 0 & 0 & 0 & 0 & 16 & 0 & 0 & 0 & 0 & 0 & 0 & 0 & 0 & 0 \\
\hline DGVM & boreal BDS & 13 & 32 & 0 & 0 & 0 & 35 & 0 & 0 & 0 & 0 & 0 & 0 & 0 & 0 & 0 & 3 & 0 & 0 & 0 & 0 \\
\hline DGVM & $\mathrm{C} 3$ & 0 & 0 & 0 & 5 & 0 & 0 & 0 & 0 & 0 & 0 & 5 & 0 & 0 & 0 & 0 & 0 & 0 & 0 & 0 & 0 \\
\hline RS & $\mathrm{BG}$ & 9 & 7 & 4 & 0 & 92 & 8 & 0 & 78 & 0 & 0 & 0 & 0 & 7 & 3 & 24 & 52 & 0 & 1 & 54 & 1 \\
\hline $\mathrm{RS}$ & boreal NET & 30 & 2 & 0 & 75 & 0 & 0 & 68 & 0 & 93 & 75 & 69 & 91 & 0 & 86 & 0 & 0 & 20 & 0 & 0 & 70 \\
\hline $\mathrm{RS}$ & temp. BDT & 6 & 0 & 0 & 6 & 0 & 0 & 15 & 0 & 0 & 2 & 7 & 1 & 0 & 0 & 0 & 0 & 0 & 0 & 0 & 1 \\
\hline $\mathrm{RS}$ & boreal BDT & 2 & 1 & 1 & 19 & 0 & 0 & 17 & 0 & 7 & 22 & 20 & 8 & 0 & 8 & 0 & 0 & 48 & 68 & 0 & 28 \\
\hline $\mathrm{RS}$ & boreal BDS & 18 & 68 & 80 & 0 & 1 & 85 & 0 & 0 & 0 & 1 & 3 & 0 & 78 & 3 & 35 & 37 & 28 & 30 & 9 & 1 \\
\hline RS & $\mathrm{C} 3$ & 35 & 23 & 14 & 0 & 7 & 7 & 0 & 22 & 0 & 0 & 1 & 0 & 16 & 0 & 41 & 11 & 3 & 0 & 37 & 0 \\
\hline $\mathrm{DM}$ & BG & 0 & 8 & 0 & 0 & 2 & 0 & 0 & 70 & 0 & 0 & 0 & 0 & 0 & 0 & 0 & 33 & 0 & 0 & 46 & 0 \\
\hline $\mathrm{DM}$ & boreal NET & 60 & 1 & 0 & 100 & 0 & 0 & 96 & 0 & 47 & 100 & 100 & 100 & 0 & 72 & 0 & 0 & 0 & 0 & 0 & 0 \\
\hline $\mathrm{DM}$ & temp. BDT & 0 & 0 & 0 & 0 & 0 & 0 & 4 & 0 & 0 & 0 & 0 & 0 & 0 & 0 & 0 & 0 & 0 & 0 & 0 & 0 \\
\hline $\mathrm{DM}$ & boreal BDT & 0 & 0 & 0 & 0 & 0 & 0 & 0 & 0 & 53 & 0 & 0 & 0 & 0 & 23 & 0 & 0 & 77 & 91 & 0 & 100 \\
\hline $\mathrm{DM}$ & boreal BDS & 40 & 91 & 100 & 0 & 0 & 100 & 0 & 3 & 0 & 0 & 0 & 0 & 100 & 4 & 100 & 63 & 23 & 9 & 54 & 0 \\
\hline $\mathrm{DM}$ & $\mathrm{C} 3$ & 0 & 0 & 0 & 0 & 98 & 0 & 0 & 26 & 0 & 0 & 0 & 0 & 0 & 0 & 0 & 4 & 0 & 0 & 0 & 0 \\
\hline AR & $\mathrm{BG}$ & 0 & 4 & 0 & 0 & 87 & 0 & 0 & 66 & 0 & 0 & 0 & 0 & 0 & 0 & 11 & 13 & 0 & 0 & 78 & 0 \\
\hline $\mathrm{AR}$ & boreal NET & 63 & 0 & 0 & 79 & 0 & 0 & 79 & 0 & 82 & 84 & 83 & 86 & 0 & 82 & 1 & 0 & 0 & 0 & 0 & 97 \\
\hline $\mathrm{AR}$ & temp. BDT & 0 & 0 & 0 & 0 & 0 & 0 & 10 & 0 & 0 & 0 & 0 & 0 & 0 & 0 & 0 & 0 & 0 & 0 & 0 & 0 \\
\hline $\mathrm{AR}$ & boreal BDT & 9 & 12 & 35 & 21 & 0 & 0 & 11 & 0 & 18 & 16 & 17 & 14 & 5 & 9 & 3 & 0 & 66 & 70 & 0 & 3 \\
\hline $\mathrm{AR}$ & boreal BDS & 28 & 75 & 63 & 0 & 0 & 99 & 0 & 10 & 0 & 0 & 0 & 0 & 87 & 9 & 79 & 83 & 34 & 30 & 18 & 0 \\
\hline AR & C3 & 0 & 9 & 1 & 0 & 13 & 1 & 0 & 25 & 0 & 0 & 0 & 0 & 8 & 0 & 6 & 5 & 0 & 0 & 3 & 0 \\
\hline
\end{tabular}




\section{Supplement S11 - DGVM spin-up and simulation of PFT profiles for each plot}

DGVM spin-up for 400 years and 20 years of simulation of PFT profiles for each of the 20 plots used in this study. For plots \#801, \#2108 and \#4268, the spin-up was extended by additional 400, 200 and 200 years respectively.

Plot405

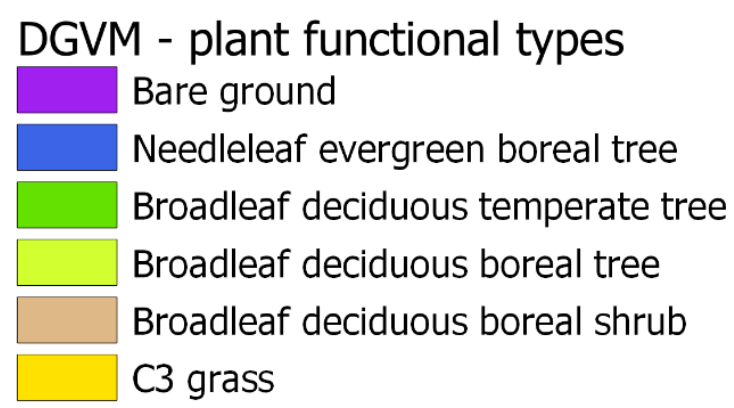

Plot513
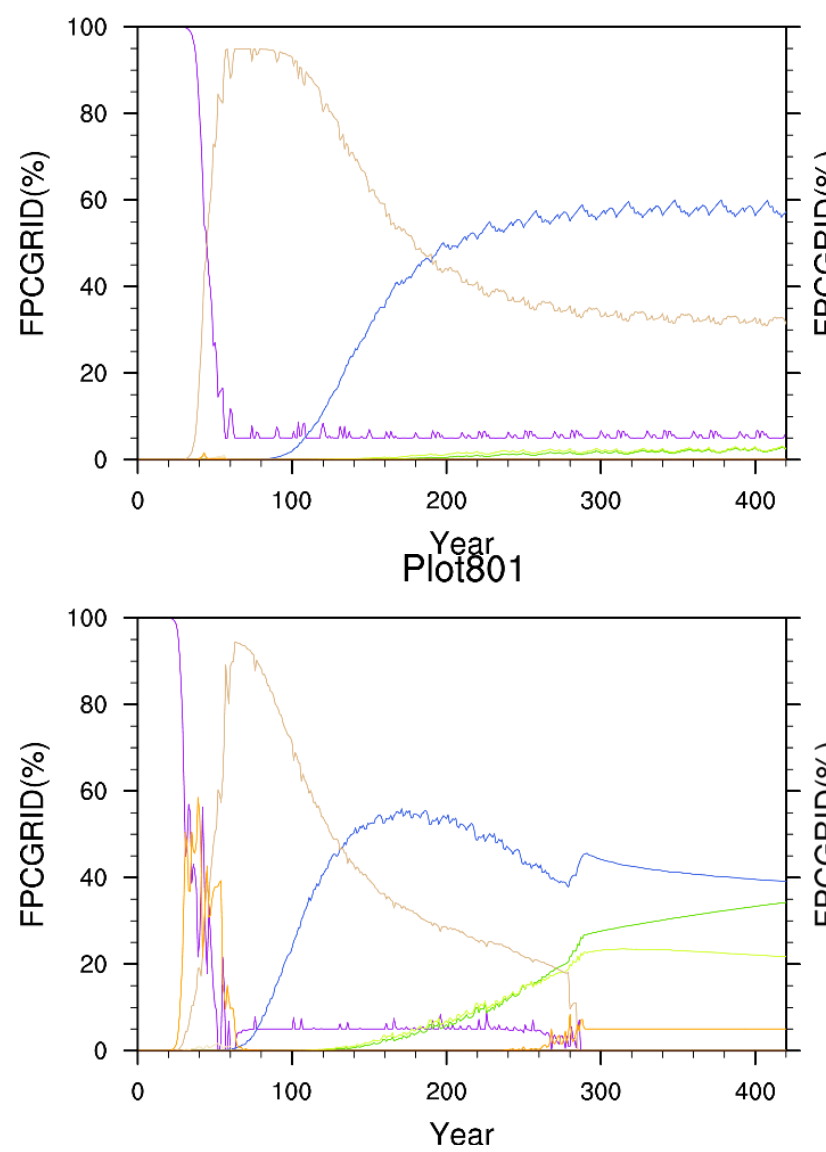
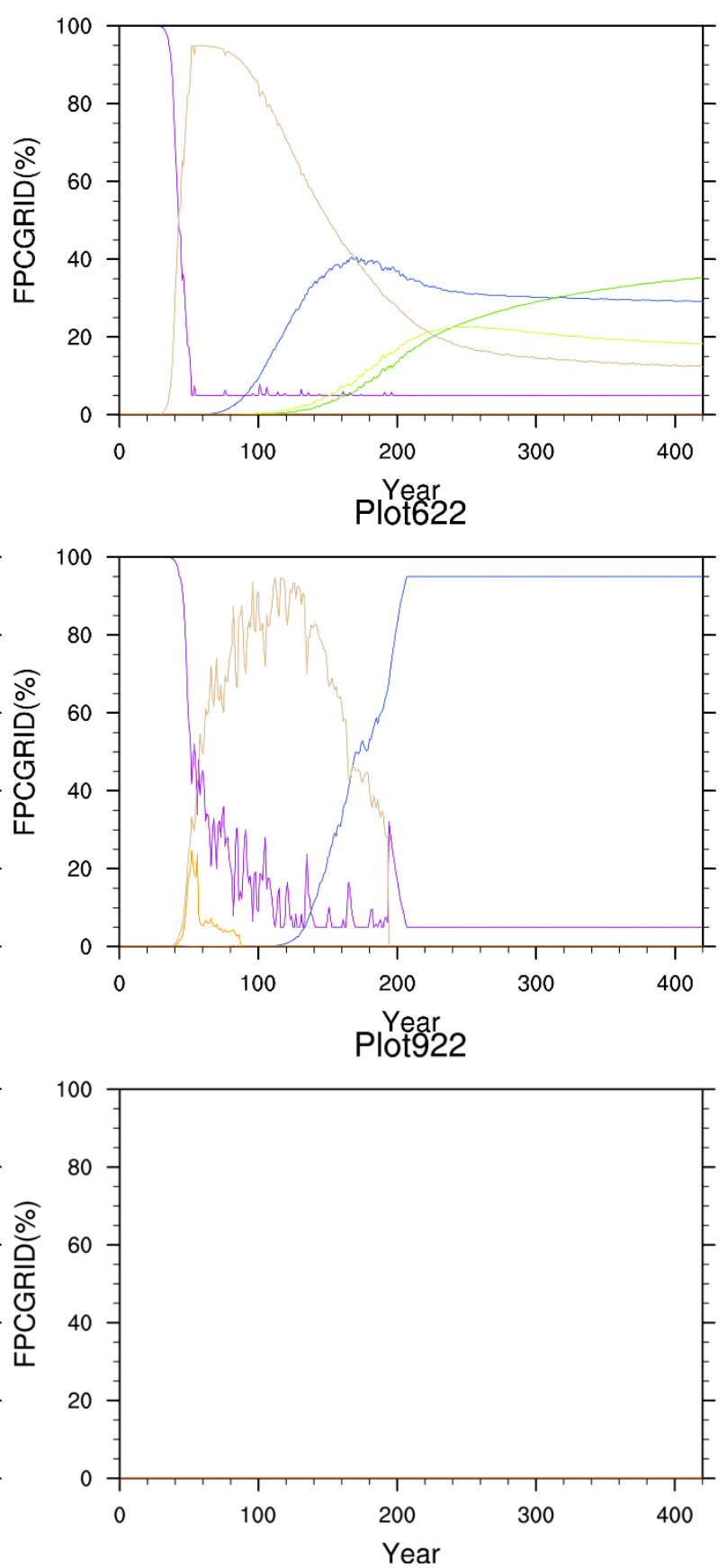
Plot1131
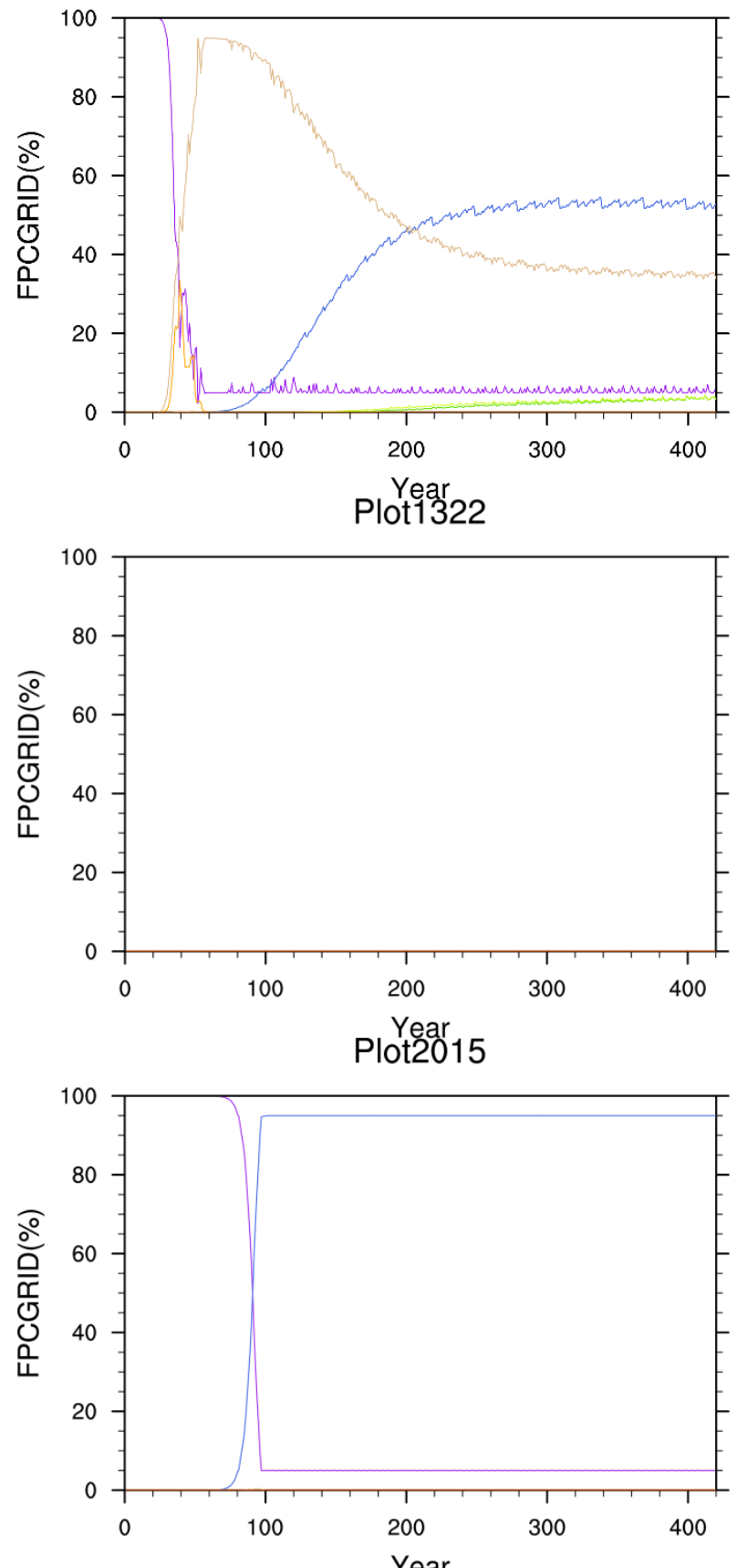

Year
Plot2238

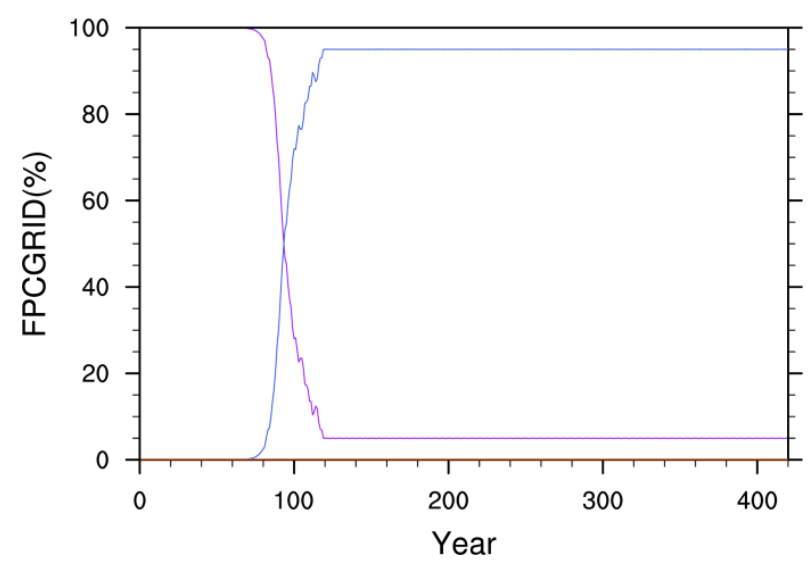

Plot1304
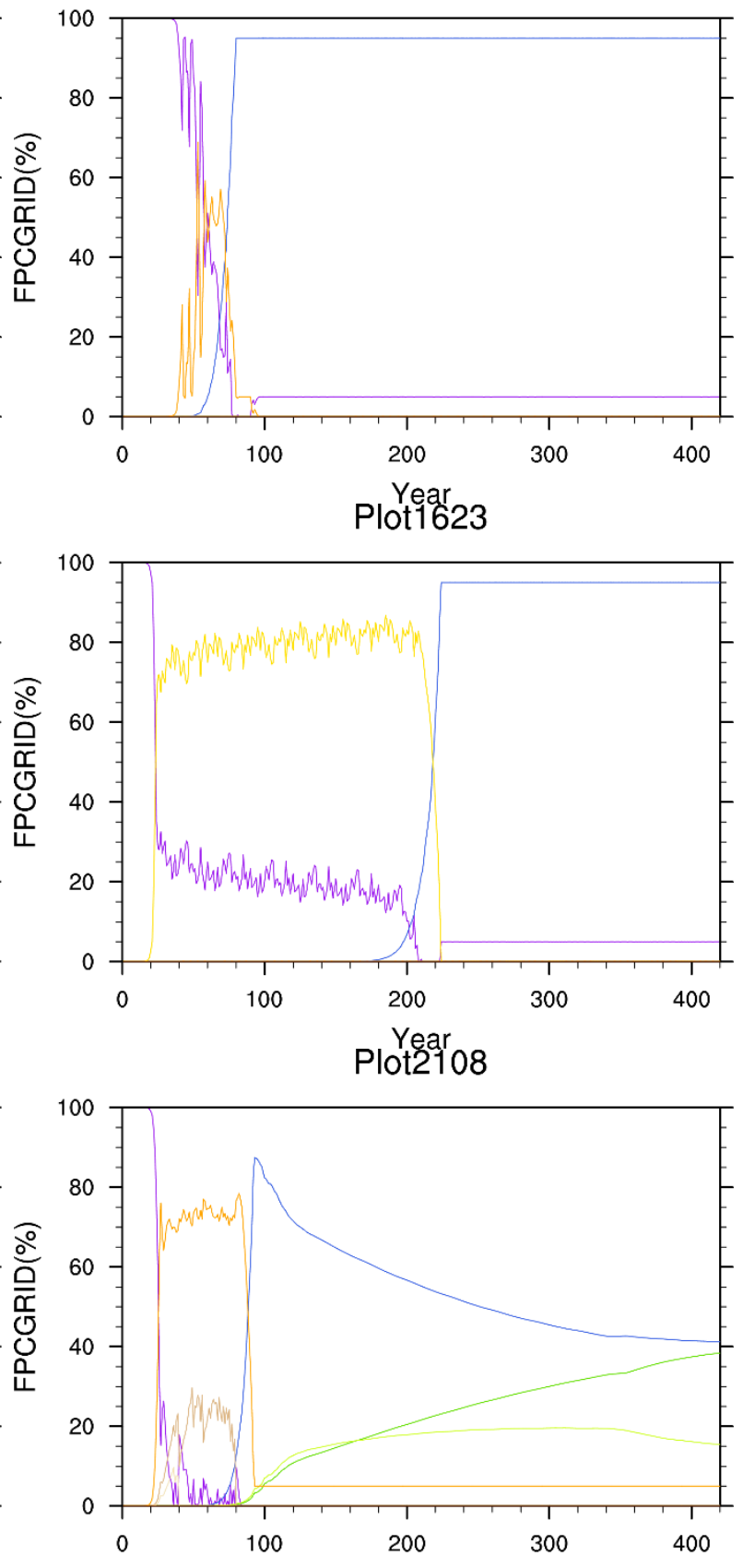

Plot2332

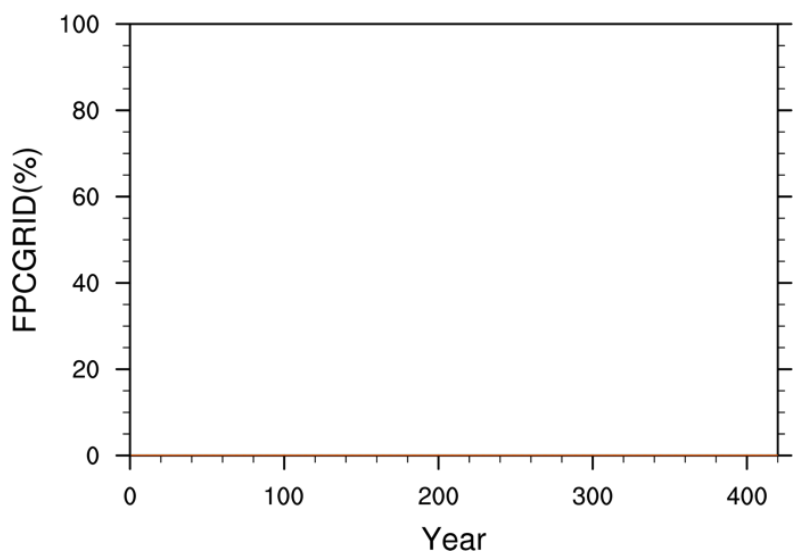


Plot2425
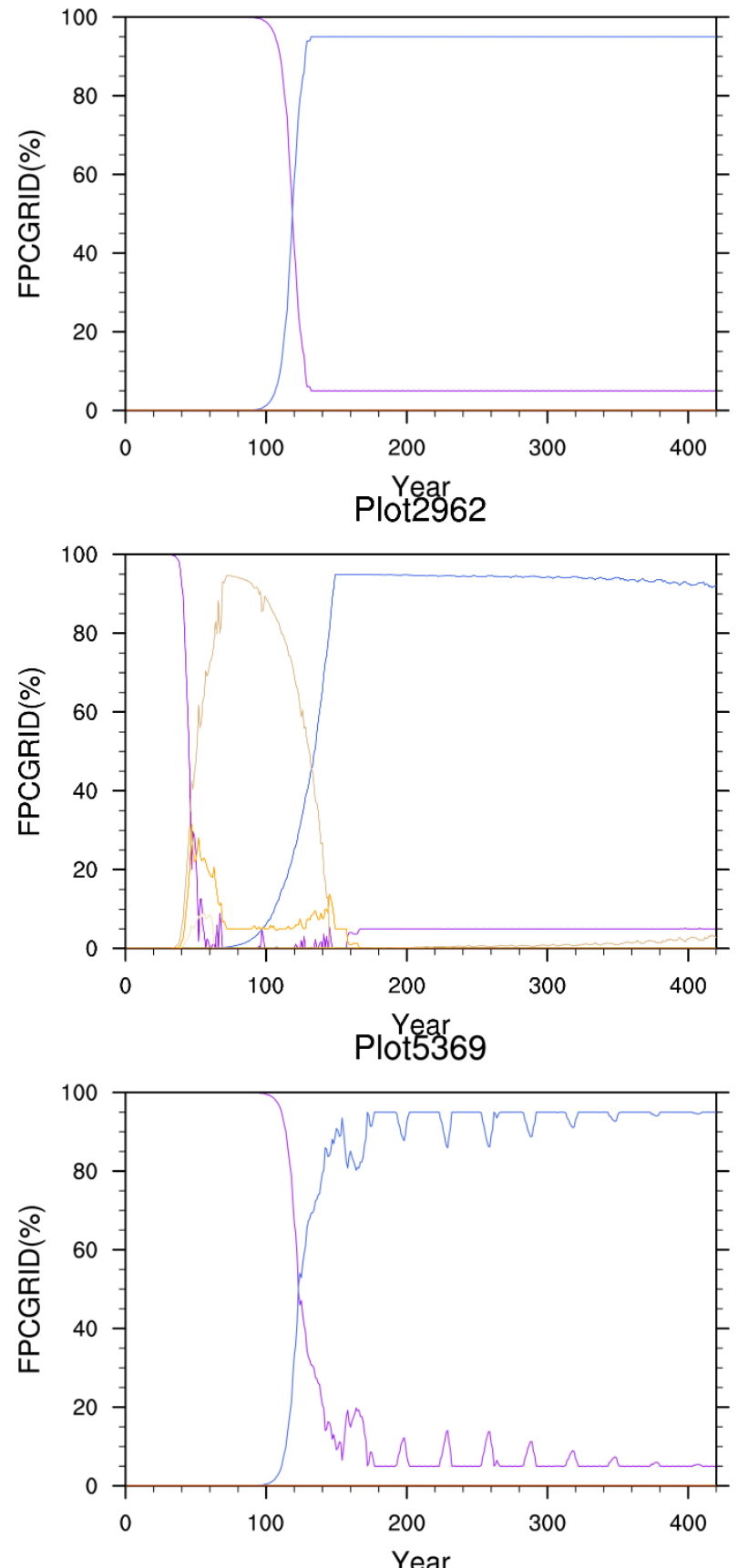

Plot6473

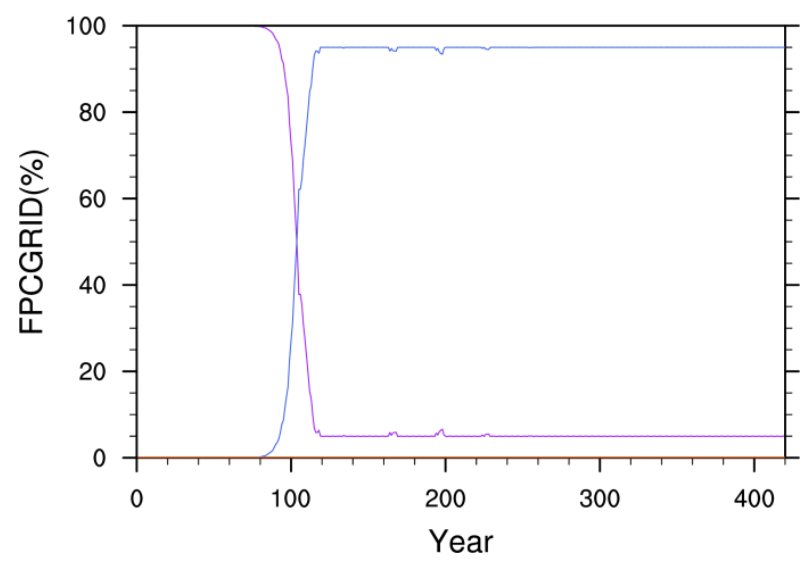

Plot2948
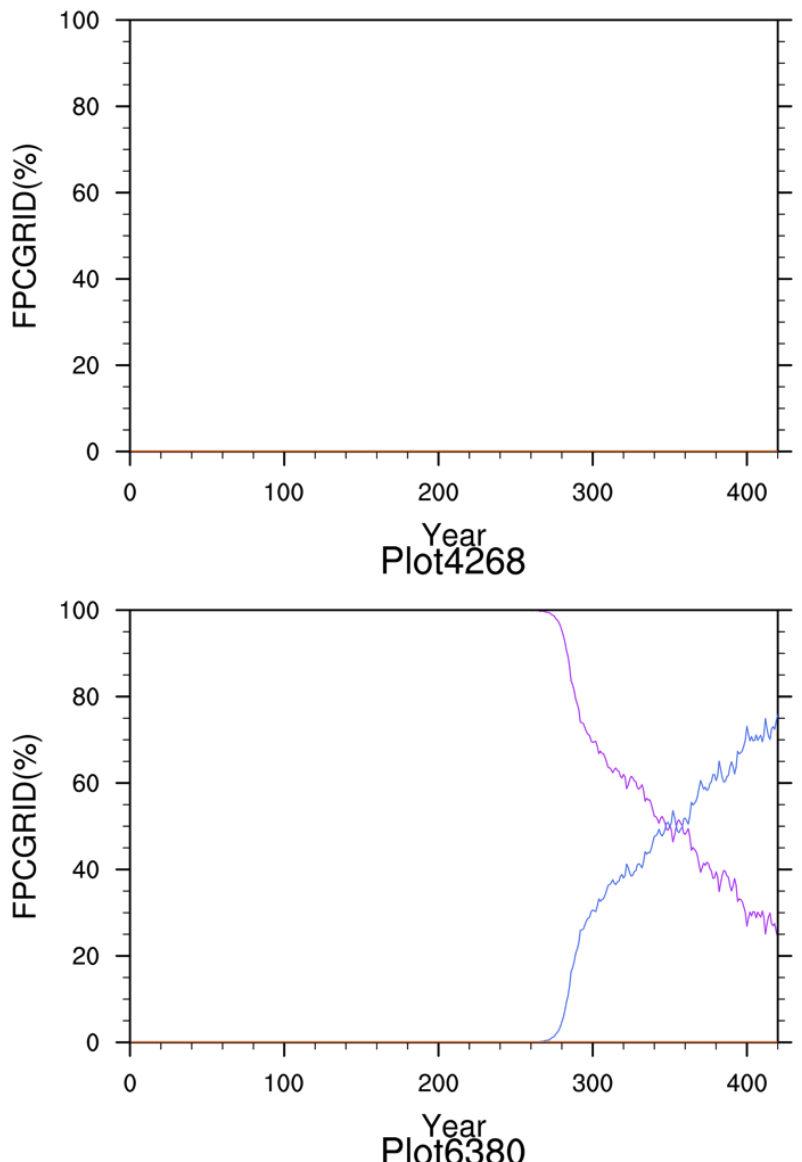

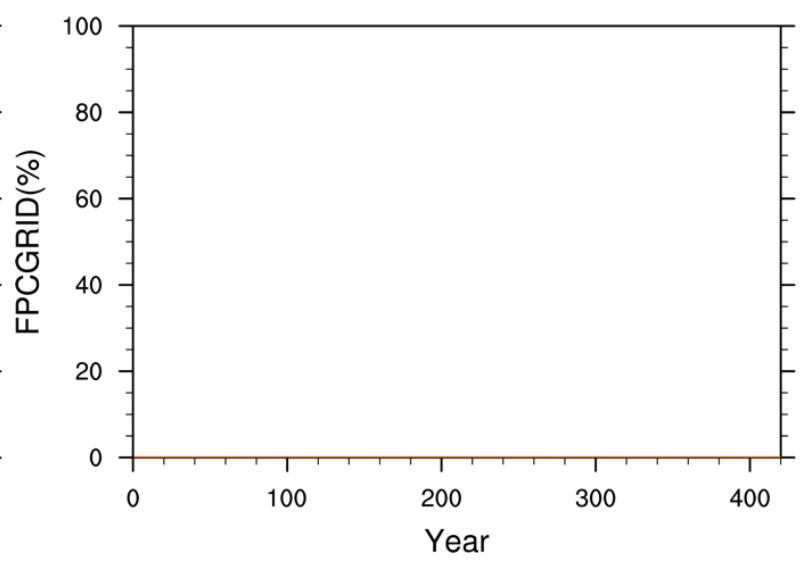


Figure S11.1 - DGVM spin-up for 400 years and simulation of PFT profiles for each of the 20 plots used in this study. FPCGRID - estimated percentage per PFT per grid cell. Reference number of plots accords with the AR18 $\times 18$ dataset, and plot numbers can be found in Table S1.

Plot801

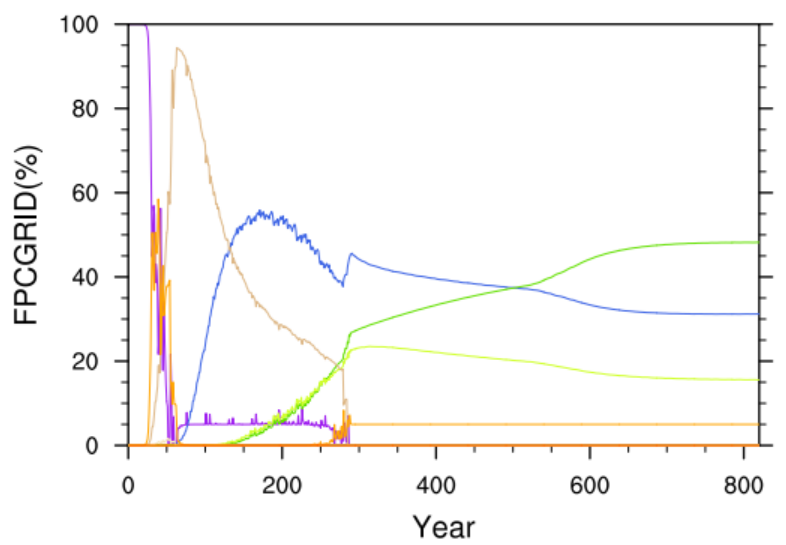

Plot4268

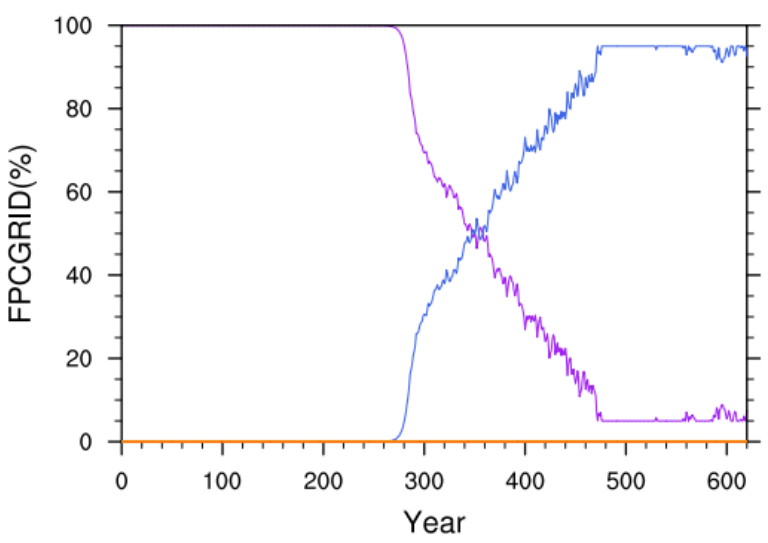

Plot2108

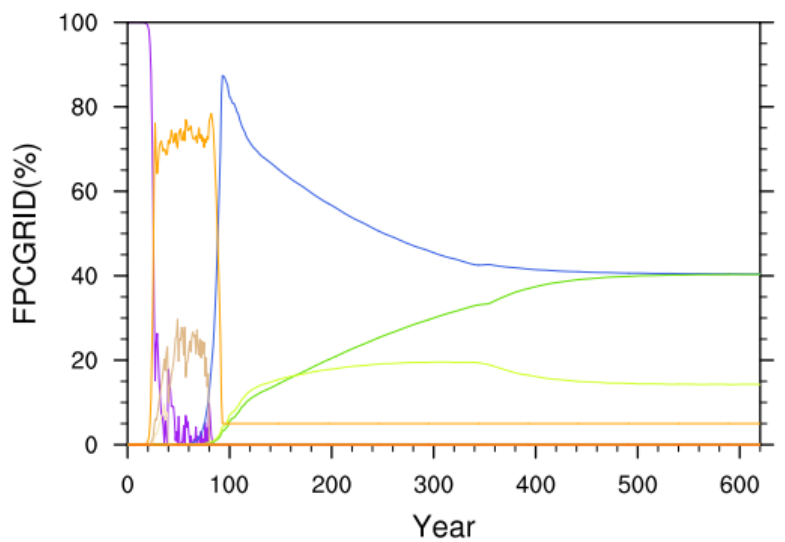

Figure S11.2 - Three plots (number 6, 12, 17) where DGVM spin-up was prolonged beyond 400 years and simulation of PFTs was extended by 400, 200 and 200 years respectively in order to check for equilibrium. FPCGRID - estimated percentage per PFT per grid cell. Reference number of plots accords with the AR18 $\times 18$ dataset, and plot numbers can be found in Table $S 1$ 


\section{Supplement S12 - Sensitivity experiments: frequency-of-presence (FoP) plots}

Frequency-of-presence (FOP) plots based upon output from distribution models (DM) for the nine combinations of three environmental variables and three vegetation types modelled, used to indicate threshold values that were explored in the sensitivity experiments, are shown in Fig. S11. Thresholds for new variables in DGVM models were chosen based upon visual inspection of the FoP plots. For example, while boreal BDS are abundant below swe_10 value of 380mm, boreal BDT and boreal NET are abundant at values of swe_10 below 180mm and 150mm respectively. Also, while we identified no clear threshold of variable bioclim_15 for boreal BDS and BDT (frequency of presence is never zero along the variable x-axis - lower left and middle panel of Fig S12), threshold for boreal NET was set to 50 (a value above which no presences occur - lower right panel of Fig S12). 
Snow water equivalent - October (swe_10)

\section{Boreal BDS $<380 \mathrm{~mm}$}

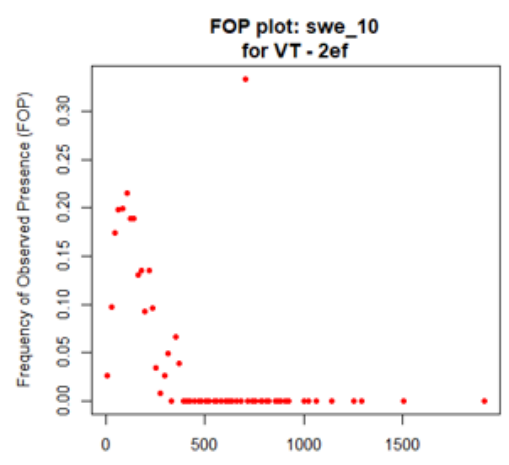

swe_10
Boreal BDT < 180mm

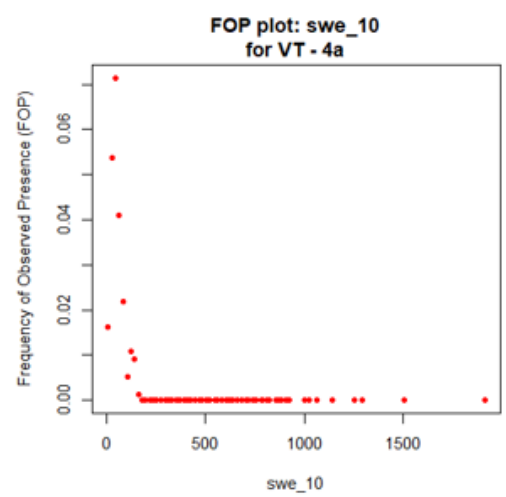

Minimum Temperature - May (tmin_5)

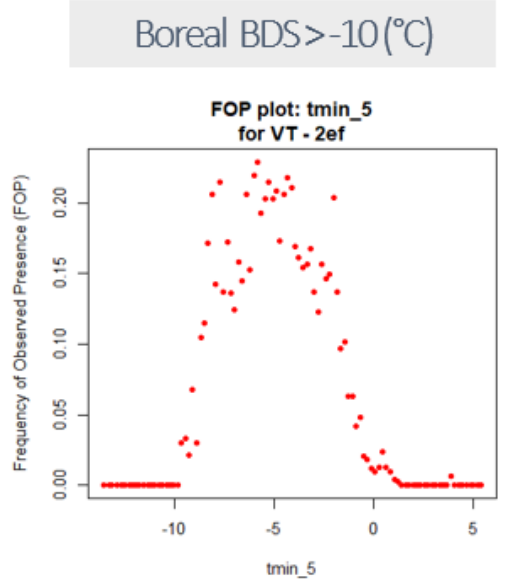

\section{Boreal BDT >-7.5 $\left({ }^{\circ} \mathrm{C}\right)$}

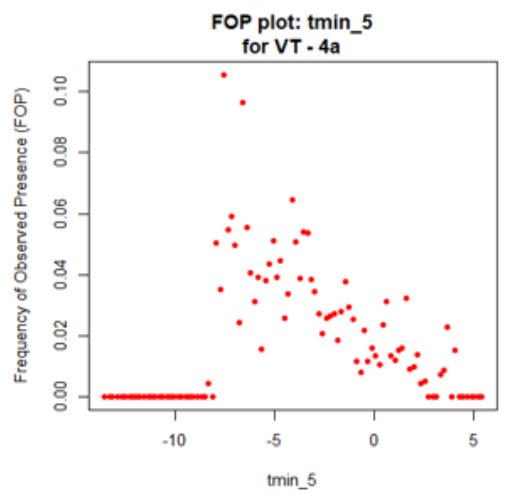

Precipitation Seasonality (bioclim_15)

\section{nothreshold for Boreal BDS}

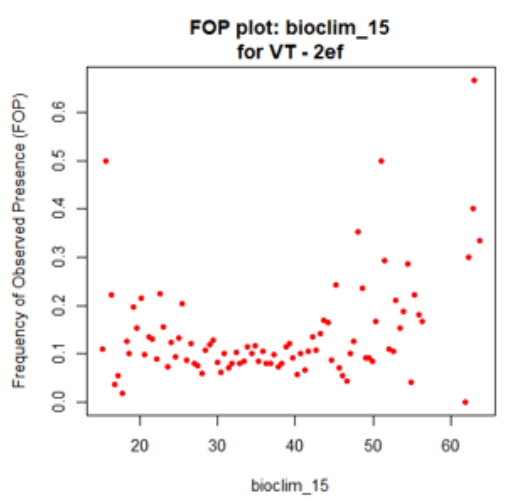

\section{no threshold for Boreal BDT}

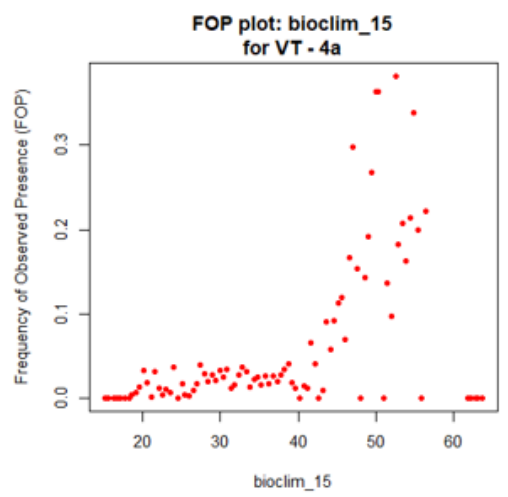

Boreal NET <150mm

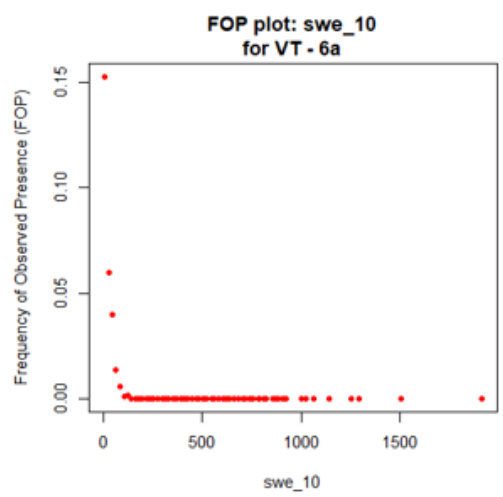

Boreal NET $>-5\left({ }^{\circ} \mathrm{C}\right)$

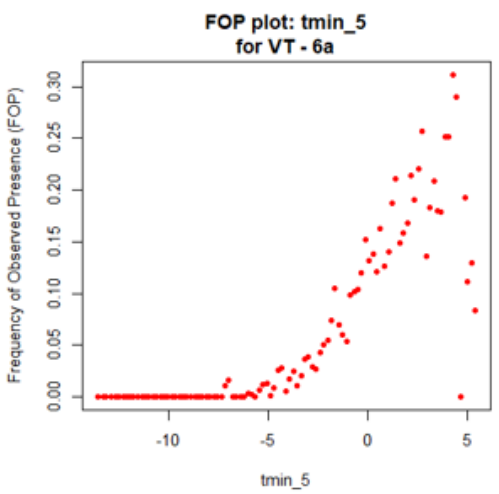

Boreal NET $<50$

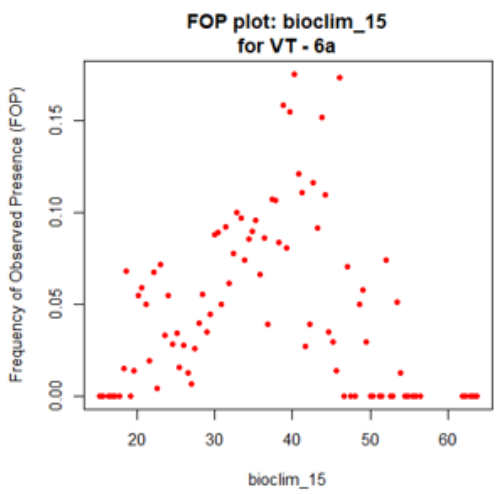

Figure S12 - Frequency-of-presence plots from the distribution modelling (DM) study by Horvath et al. (2019) for the combinations of environmental predictors and vegetation types (VTs) used in the sensitivity experiments with DGVM. FOP is the frequency of $100 \times 100 \mathrm{~m}$ pixels in the $\mathrm{AR} 18 \times 18$ dataset in which the VT in question is present, expressed as a fraction of all pixels in that interval along the environmental variable. All environmental variables were a priori divided into 100 intervals with the same number of pixels. The environmental gradients were: swe_10 - snow water equivalent in October $(\mathrm{mm})$; $\operatorname{tmin} \_5$-- minimum temperature in May $\left({ }^{\circ} \mathrm{C}\right)$; bioclim_15 - precipitation seasonality (unitless index). Boreal BDS - boreal broadleaf deciduous shrubs, Boreal BDT - boreal broadleaf deciduous trees, Boreal NET - boreal needleleaf evergreen shrubs. 


\section{Supplement S13 - Sensitivity experiments: results}

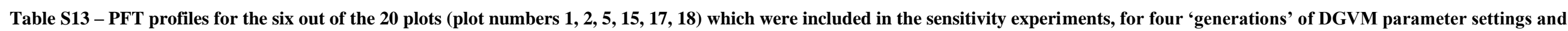

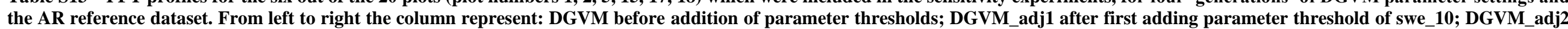

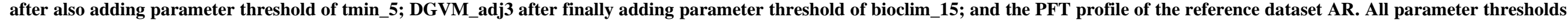
were added cumulatively. Full names for the PFTs are given in Table S7 and names of parameters and their values in Table 3.

\begin{tabular}{|c|c|c|c|c|c|c|c|c|c|c|c|c|c|c|c|c|c|c|c|c|c|c|c|c|c|c|c|c|c|c|}
\hline & 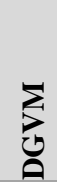 & 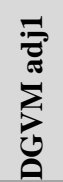 & 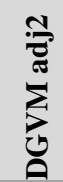 & 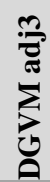 & $\dddot{z}$ & $\sum_{0}^{\sum_{0}}$ & $\sum_{\substack{5\\
}}^{:}$ & 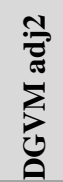 & 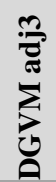 & $\dddot{z}$ & 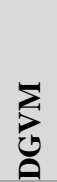 & 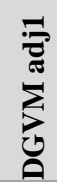 & 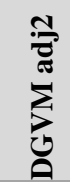 & 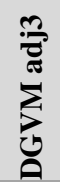 & $\dddot{z}$ & 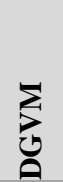 & 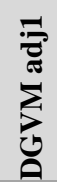 & 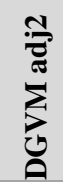 & 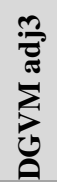 & 爻 & $\sum_{\substack{0 \\
0}}$ & 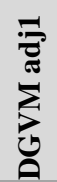 & 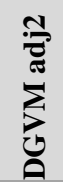 & 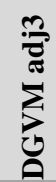 & $\dddot{y}$ & $\sum_{0}^{\sum}$ & 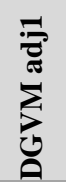 & 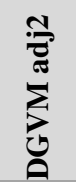 & 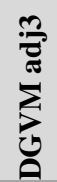 & $\frac{x}{4}$ \\
\hline & \multicolumn{5}{|c|}{ plot 1} & \multicolumn{5}{|c|}{ plot 2} & \multicolumn{5}{|c|}{ plot 5} & \multicolumn{5}{|c|}{ plot 15} & \multicolumn{5}{|c|}{ plot 17} & \multicolumn{5}{|c|}{ plot 18} \\
\hline BG & 5 & 5 & 5 & 9 & 0 & 6 & 5 & 5 & 5 & 4 & 6 & 6 & 6 & 7 & 0 & 5 & 5 & 5 & 3 & 13 & 28 & $\begin{array}{c}10 \\
0\end{array}$ & $\begin{array}{c}10 \\
0\end{array}$ & $\begin{array}{c}10 \\
0\end{array}$ & 0 & 5 & $\begin{array}{c}10 \\
0\end{array}$ & $\begin{array}{c}10 \\
0\end{array}$ & $\begin{array}{c}10 \\
0\end{array}$ & 0 \\
\hline $\begin{array}{c}\text { boreal } \\
\text { NET }\end{array}$ & 95 & 95 & 95 & 0 & 0 & 58 & 58 & 58 & 0 & 0 & 52 & 52 & 52 & 0 & 0 & 92 & 92 & 92 & 0 & 0 & 72 & 0 & 0 & 0 & 0 & 95 & 0 & 0 & 0 & 0 \\
\hline $\begin{array}{l}\text { temp. } \\
\text { BDT }\end{array}$ & 0 & 0 & 0 & 0 & 0 & 2 & 2 & 2 & 33 & 0 & 4 & 4 & 4 & 13 & 0 & 0 & 0 & 0 & 1 & 0 & 0 & 0 & 0 & 0 & 0 & 0 & 0 & 0 & 0 & 0 \\
\hline $\begin{array}{c}\text { boreal } \\
\text { BDT }\end{array}$ & 0 & 0 & 0 & 0 & 35 & 2 & 2 & 2 & 31 & 12 & 4 & 4 & 4 & 13 & 0 & 0 & 0 & 0 & 2 & 0 & 0 & 0 & 0 & 0 & 66 & 0 & 0 & 0 & 0 & 70 \\
\hline $\begin{array}{c}\text { boreal } \\
\text { BDS }\end{array}$ & 0 & 0 & 0 & 91 & 63 & 32 & 32 & 32 & 31 & 75 & 35 & 35 & 35 & 67 & 99 & 3 & 3 & 3 & 89 & 83 & 0 & 0 & 0 & 0 & 34 & 0 & 0 & 0 & 0 & 30 \\
\hline C3 & 0 & 0 & 0 & 0 & 1 & 0 & 0 & 0 & 0 & 9 & 0 & 0 & 0 & 0 & 1 & 0 & 0 & 0 & 6 & 5 & 0 & 0 & 0 & 0 & 0 & 0 & 0 & 0 & 0 & 0 \\
\hline
\end{tabular}

